\title{
Functional Appliances - a review and presentation of four cases.
}

SADJ September 2017, Vol 72 no 8 p372 - p378

NN Desai', SM Dawjee ${ }^{2}$

\section{INTRODUCTION}

Orthodontic treatment is aimed at improving facial and dental appearances as well as enhancing the relationships of the teeth and skeletal bases to each other. As orthodontic treatment is becoming more accessible, parents and caregivers are requesting attention for their children at an earlier age. The goal of early treatment is to correct existing or developing skeletal, dentoalveolar and muscular imbalances.

There are a number of modalities available for managing Class II malocclusions. Some of the more common options include extra-oral traction appliances, arch expansion appliances, extraction procedures, functional jaw orthopaedic appliances and orthognathic surgery. The treatment approach adopted will depend on the growth status of the patient. Treatments that have the ability to alter a patient's facial growth exert their effect, either accelerating or limiting, on the skeletal structures of the craniofacial region.

The concept of functional jaw orthopaedics or advancement of the mandible is not new to dentistry. As cited by Graber ${ }^{4}$, Norman Kingsley was the first to use forward posturing of the mandible by "jumping the bite". He developed a maxillary vulcanite plate that guided the mandible into a forward position during mandibular closure.

In 1902, Pierre Robin introduced the plastic monobloc as a passive positioning device. This appliance influenced the muscular activity by a change in the spatial relationship of the jaws. It was specifically designed to act on the maxillary and the mandibular arches simultaneously with the intention of expanding the upper and lower arches to bring the mandible forward. ${ }^{5}$ Since then, numerous appliances have been designed and developed with the intention to advance and reposition the mandible.

There has also been a gradual evolution in the way functional jaw orthopaedics is used in a contemporary

1. Nitinkumar Desai: $B C h D(U W C), P D D(O r t h o)(U W C), M C h D$ (Ortho) (UP). Consultant: Department of Orthodontics, School of Oral Health Sciences, University of Pretoria.

2. SM Dawjee: BChD, BChDHons, MSc(Odont), MDent(Ortho), PGDip/RE, PhD. Head of Department: Department of Orthodontics, School of Oral Health Sciences, University of Pretoria.

\section{Corresponding author}

Nitinkumar Desai:

Consultant: Department of Orthodontics, University of Pretoria, P.O. Box 1266, Pretoria, 0001. Tel: 012319 2306/2510, Cell: 082777 0045, Fax: 012328 6697/ 086622 4420. E-mail: Nitinkumar.Desai@up.ac.za orthodontic practice, especially with regard to appliance selection, the timing of intervention and the urgency and need for "orthopaedic" correction. These functional appliances were developed to correct the aberrant muscle environment - the jaw-to-jaw relationship - and as a result restore facial balance by improving function. ${ }^{1}$

The timing of early treatment is pivotal in the modification of facial growth. Ball et al. conducted a study in 2011 with the intention to establish the relationship of mandibular growth to the stages of cervical vertebral maturation. They found that peak mandibular growth velocity occurred most frequently during stage four. This stage is defined by concavities at the lower borders of C2, C3, and C4 with the bodies of both C3 and $\mathrm{C} 4$ being horizontally rectangular in shape. Ball et al. found the average number of years spent in this stage to be 3.79 years and the average amount of mandibular growth occurring during stage four to be $9.40 \mathrm{~mm} .^{1}$

In an attempt to demonstrate the potential benefit of early functional treatment, four cases treated at the Oral and Dental Hospital of the University of Pretoria are presented.

\section{CASE REPORT 1}

A 10-year-old female patient (Figures 1a-h) presented with the complaint that her teeth were crooked and that she found it difficult to chew. The patient requested an improvement in her appearance, wanting her teeth to be straight and an improvement in her function so that she could eat and chew more effectively.

Clinically the patient presented with a Class II Division One malocclusion on a moderate Class II skeletal base with mandibular retrognathia. The patient was in the transitional dentition stage of dental development with permanent incisors and molars present together with the primary molars and canines.

In occlusion the overbite was $7 / 10$ ths (70\%) and the overjet was $10 \mathrm{~mm}$. The molar relationship was Class II bilaterally. The cephalometric tracing confirmed that the patient had a moderate Class II skeletal base relationship with an ANB of $9^{\circ}$ and the mandibular plane angle of $20.7^{\circ}$. The upper incisors were slightly proclined at $23^{\circ}$ with the lower incisors retroclined at $22^{\circ}$ (norm values; $22^{\circ}$ and $25^{\circ}$. The interincisal angle was $125^{\circ}$.

This patient was treated with a Twin Block appliance ${ }^{2}$ which essentially consists of upper and lower removable bite planes which meet on an incline when the patient occludes, producing a protrusion of the lower jaw. Initially 

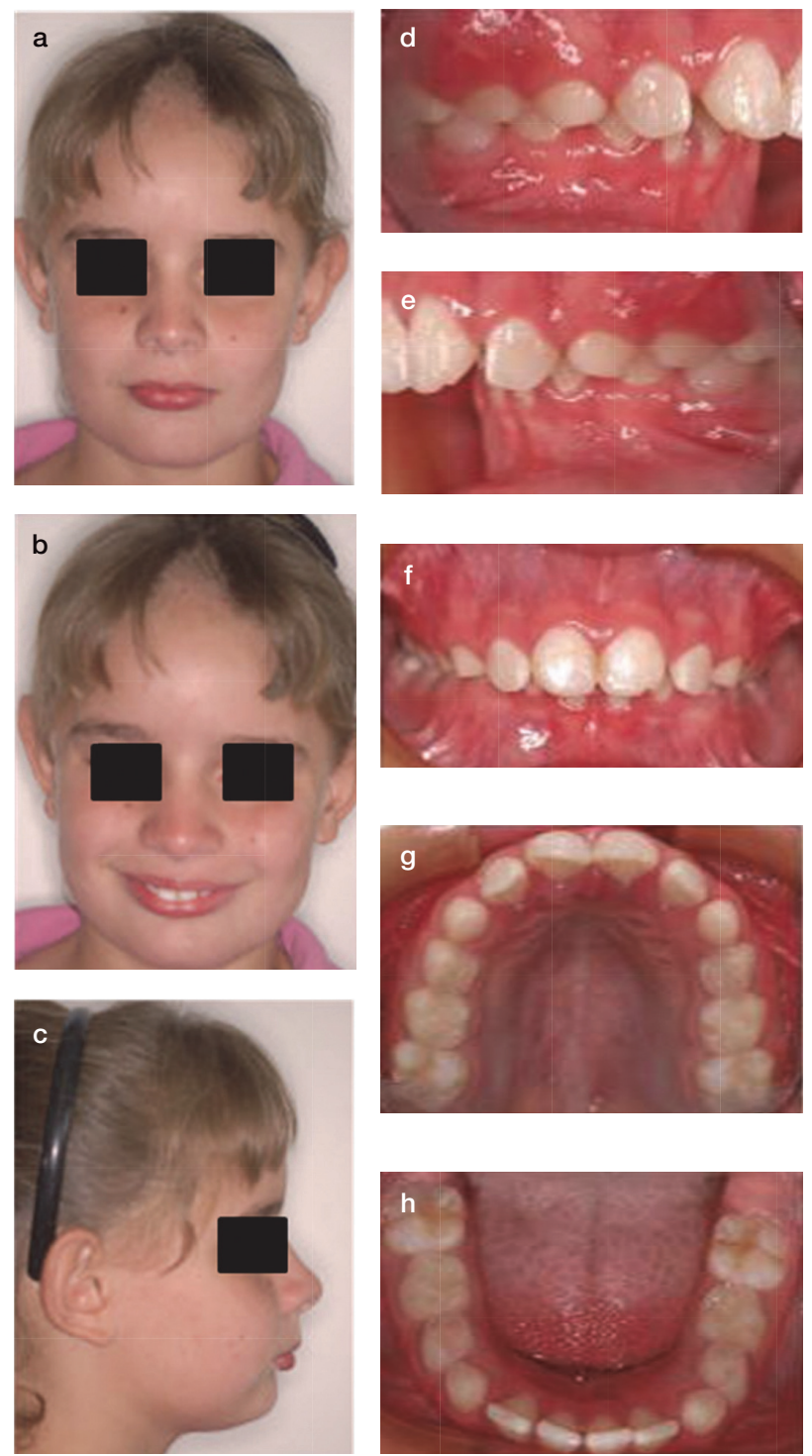

Figure 1 (a-h): Case Report 1, pre-treatment photographs.

a 2x4 appliance was used for three months to level and align the anterior maxillary segment. This was followed by the placement of removable Twin Block appliances and the patient was instructed on the wear and care of the appliance.

\section{DIGITIZED LATERAL CEPH, PRE-TREATMENT}

DIGITIZED LATERAL CEPH, END OF PHASE-1

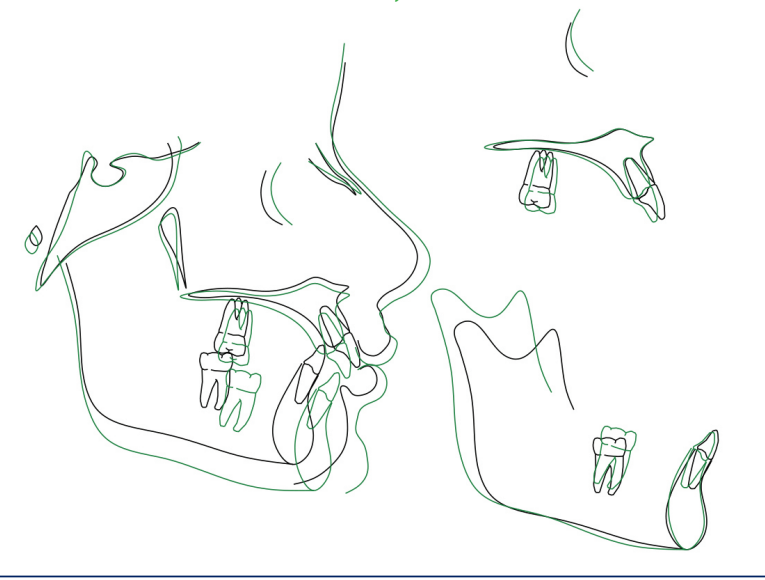

Figure 3: Case Report 1, Ricketts Superimposition - End of Phase I.
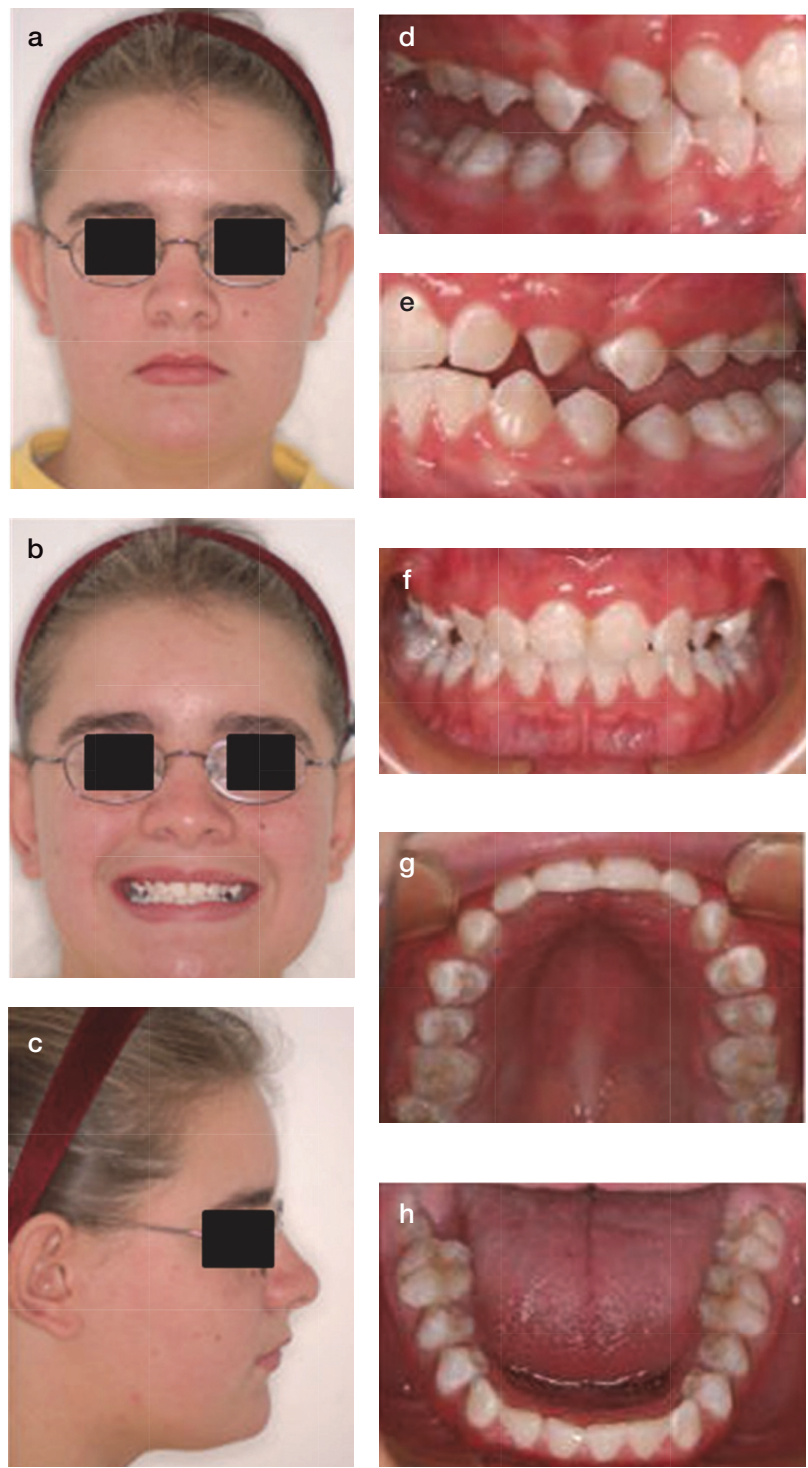

Figure 2 (a-h): Case Report 1, end of Phase-I photographs.

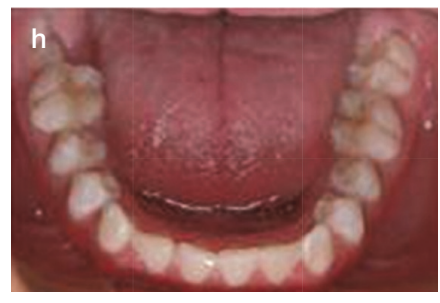

The Twin Block appliances were trimmed as the succedaneous teeth erupted and the buccal blocks were trimmed posteriorly to allow for the eruption of the first molars. The appliances were worn full time for 20,5 months followed by night time wear only for another eight months (Figures 2a-h).

Following this period of treatment, the antero-posterior correction of the buccal segments was achieved, the upper incisors had been retroclined by $8^{\circ}$, the lower incisors proclined by $8^{\circ}$ and the ANB angle was reduced by $4,5^{\circ}$. Superimposition of cephalometric tracings revealed mandibular changes in a horizontal and vertical direction, with a favourable increase in lower anterior face height of $3.5 \mathrm{~mm}$ (Figure 3).

\section{CASE REPORT 2}

A 14-year-old male (Figures 4a-h) presented with a concern about the appearance of his upper front teeth. He presented with a Class II Division Two incisor relationship on a skeletal Class II base with a retrognathic mandible. He had a full complement of teeth with moderate crowding of the upper labial segment. 
In occlusion the overbite was 8/10ths (80\%) and the overjet was $5 \mathrm{~mm}$. The buccal segment relationship was a full unit Class II bilaterally. The cephalometric tracing confirmed that the patient had a skeletal Class II base relationship with an ANB of $6^{\circ}$. The mandibular plane angle was $23^{\circ}$. The upper incisors were retroclined at $17^{\circ}$ with the lower incisors at a correct inclination at $25^{\circ}$. The interincisal angle was $132^{\circ}$.

Treatment involved correcting the Class II skeletal relationship with a Twin Block appliance. Removable Twin Block appliances were fitted and instructions were given on wear and care of the appliances. The patient was monitored and the bite blocks were trimmed posteriorly to allow for molar eruption. After 6,5 months of full time wear the patient was instructed to wear the appliance at night only as a retentive measure. Interim results were reassuring and there has been a change in mandibular position (Figures 5a-h). Treatment is ongoing and the patient will be evaluated for the placement of full fixed appliances in three months.

\section{CASE REPORT 3}

A 14-year-old male (Figures 6a-h) presented with a complaint that his "top teeth are too far forward." He
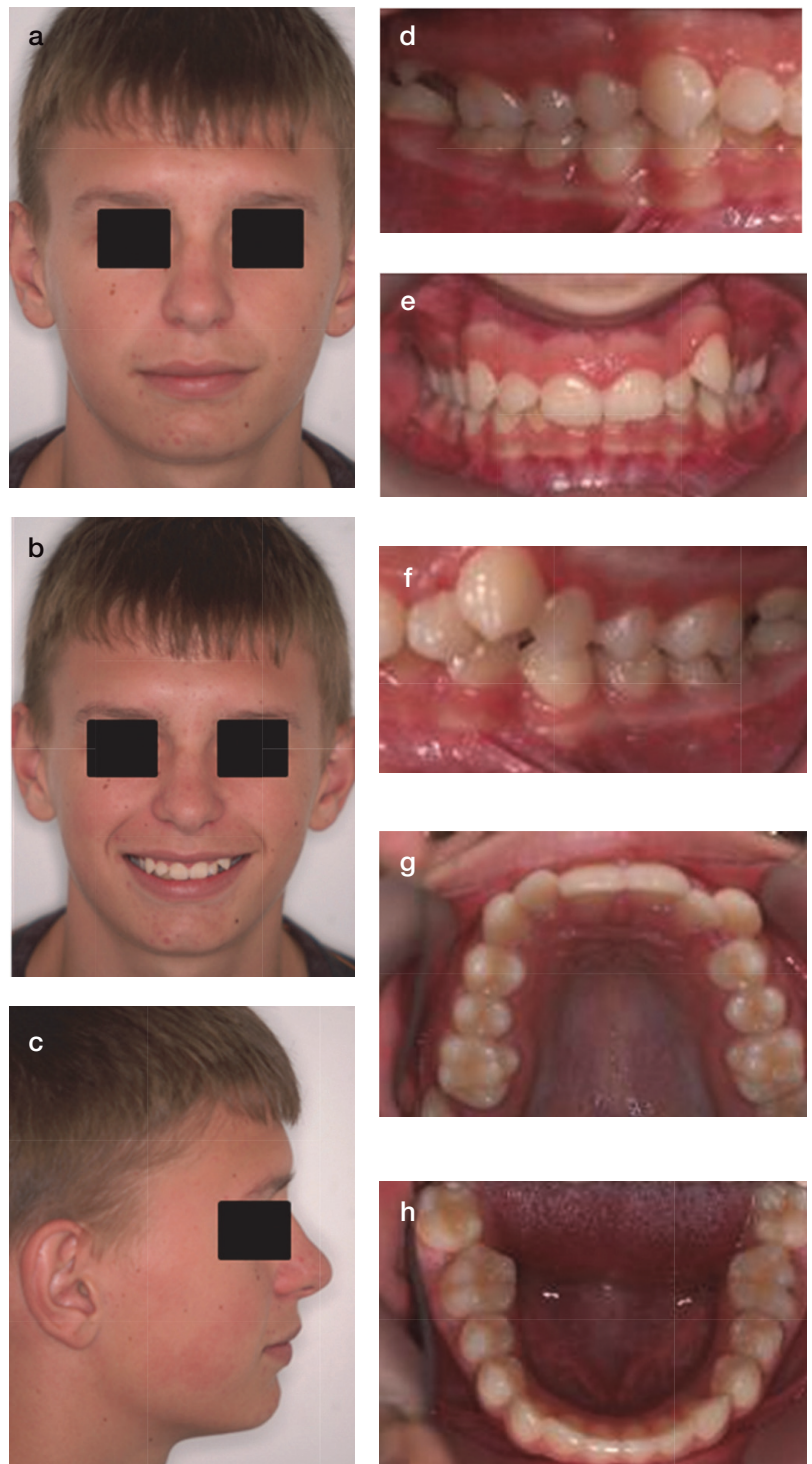

Figure 4 (a-h): Case Report 2, pre-treatment photographs.

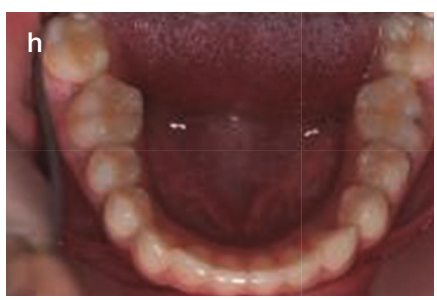

presented with a Class II Division One incisal relationship on a Class II skeletal base with mandibular retrognathia. He had a full complement of teeth with mild crowding of the upper arch and severe crowding of the lower arch. In occlusion the overbite was 8/10ths and the overjet was 10 $\mathrm{mm}$. The buccal segment relationship was a full unit Class II bilaterally. The cephalometic tracing confirmed that the patient had a skeletal Class II base relationship with an ANB angle of $6^{\circ}$. The mandibular plane angle was $25^{\circ}$. The upper incisors were proclined at $35^{\circ}$ with the lower incisors showing a compensatory proclination of $29^{\circ}$. The inter-incisal angle was $113^{\circ}$.

The aim of treatment was to achieve sagittal correction with the Mandibular Anterior Repositioning Appliance (MARA) appliance. ${ }^{3}$ Fixed orthodontic appliances were bonded in the upper and lower arches from second molar to second molar after extraction of the upper and lower first premolars (14, 24, 34 \& 44). Thirteen months after bonding the MARA was placed to advance the mandible by $4 \mathrm{~mm}$ on the left and $5 \mathrm{~mm}$ on the right. During follow-up appointments, the lower jaw was advanced incrementally and asymmetrically to achieve an edge-to-edge bite with coincidental midlines. After seven months, TMJ radiographs were taken to confirm that the condyles were centred in the fossae and subsequently the MARA elbows were removed. Two
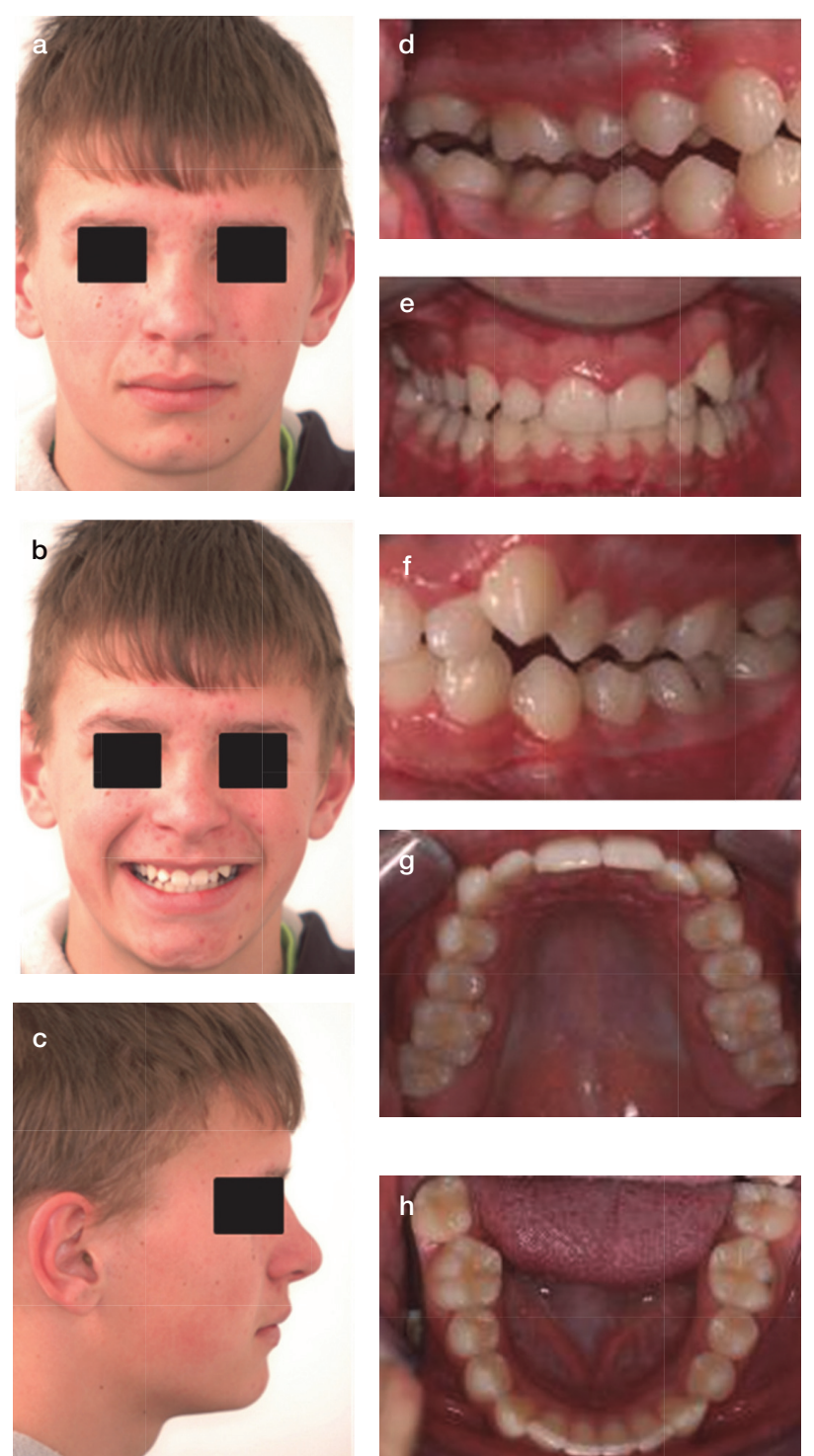

Figure 5 (a-h): Case Report 2, interim photographs. 

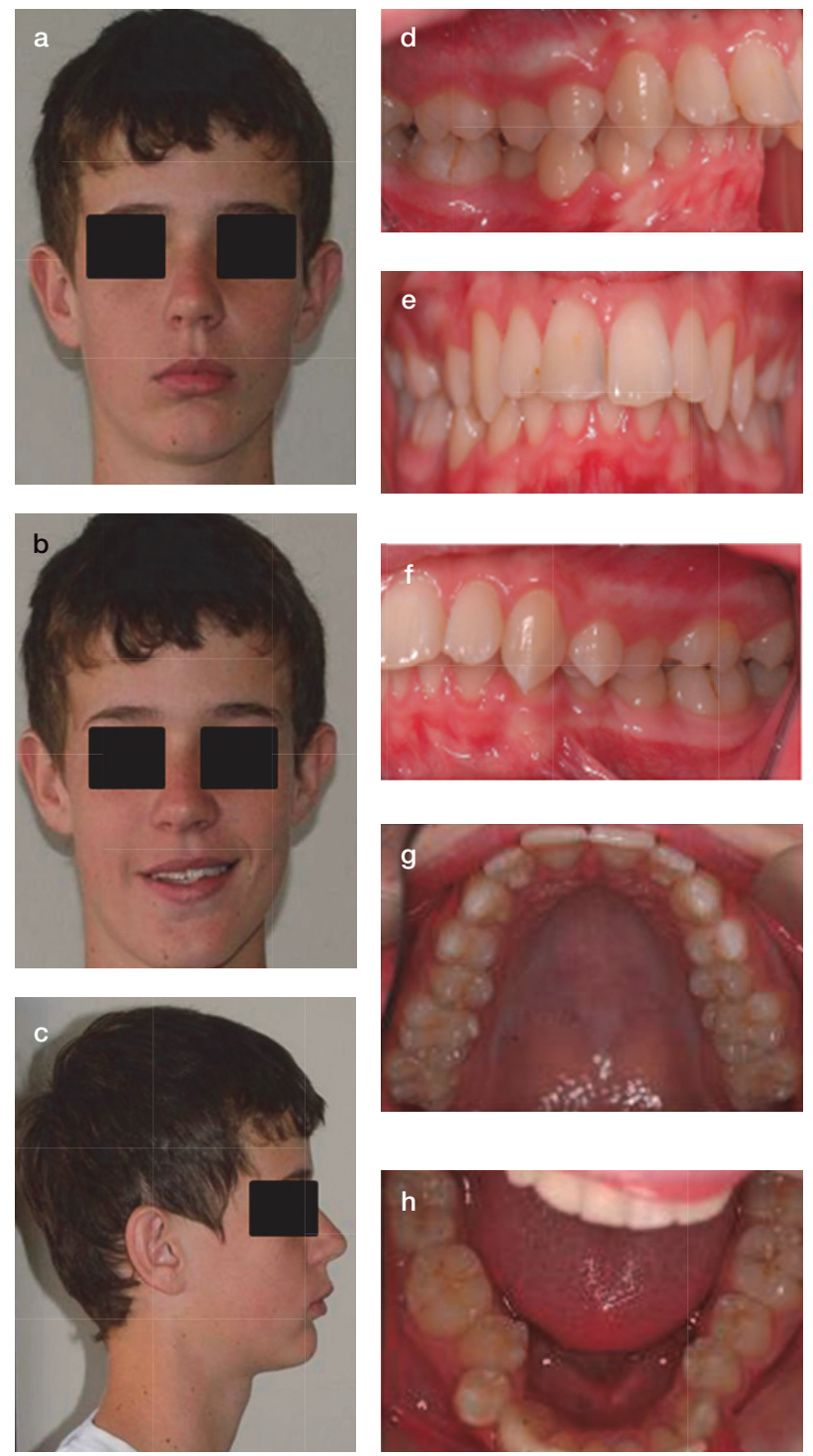

Figure 6 (a-h): Case Report 3, pre-treatment photographs.

weeks later, the remaining components of the MARA were removed. Final detailing was done to produce a mutually protected functional occlusion. Total treatment time was 27 months (Figures 7a-h).

Cephalometric analysis revealed that the sagittal correction occurred due to an anterior repositioning

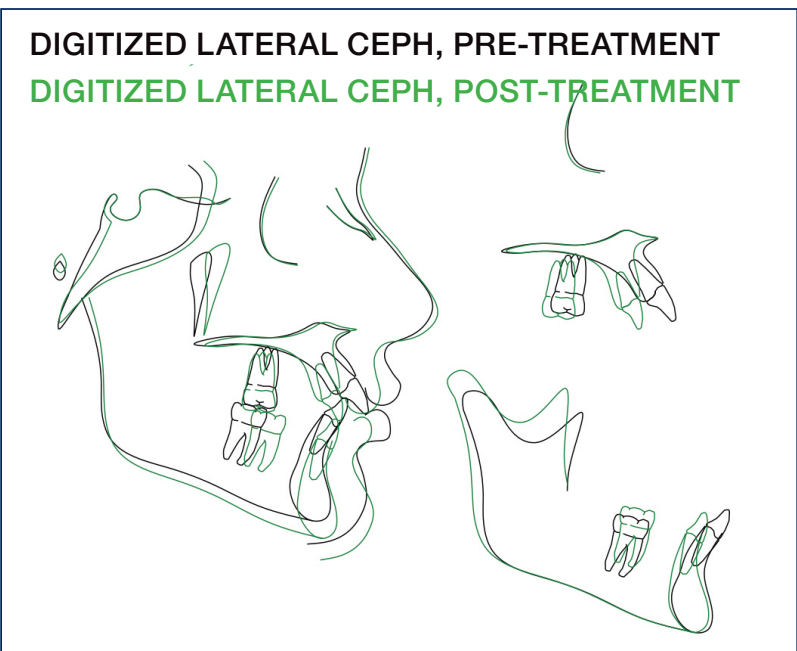

Figure 8: Case Report 3, Ricketts Superimposition - Post-treatment.
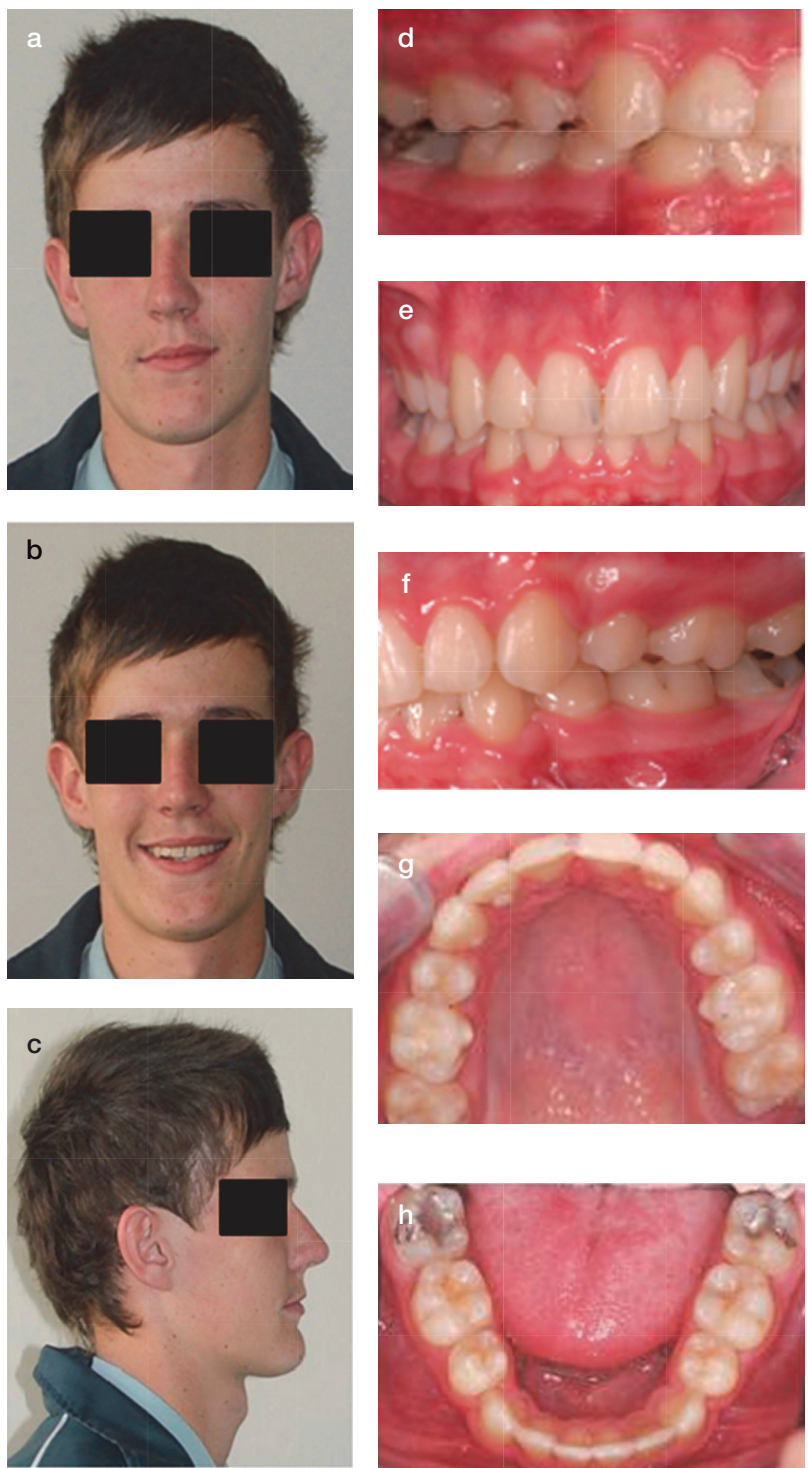

Figure 7 (a-h): Case Report 3, post-treatment photographs.

of B point with no evidence of maxillary restraint. The upper labial segment was normalized at $22^{\circ}$, the lower retroclined to $20^{\circ}$ and an interincisal angle of $138^{\circ}$ was achieved (Figure 8).

\section{CASE REPORT 4}

A 13-year-old female (Figures 9a-h) presented with a complaint that her teeth were 'crooked'. She had a Class II Division Two incisor relationship on a skeletal Class II base with a retrognathic mandible. A full complement of teeth was present with moderate crowding of the upper and lower labial segments. In occlusion the overbite was 8/10ths and the overjet was $3 \mathrm{~mm}$. The buccal segment relationship was Class II bilaterally. The cephalometric tracing confirmed that the patient had a skeletal Class II base relationship with an ANB of $6^{\circ}$. The mandibular plane angle was $30^{\circ}$. The upper incisors were retroclined at $7^{\circ}$ as were the lower incisors at $17^{\circ}$. The interincisal angle was $151^{\circ}$.

Treatment involved correcting the Class II skeletal relationship with a MARA appliance. Fixed orthodontic appliances were bonded in the upper arch 4-4 and the lower arch 5-5. Two weeks later the MARA appliance was added to advance the mandible by $3 \mathrm{~mm}$ on the left and $4 \mathrm{~mm}$ on the right. During follow-up appointments, the 

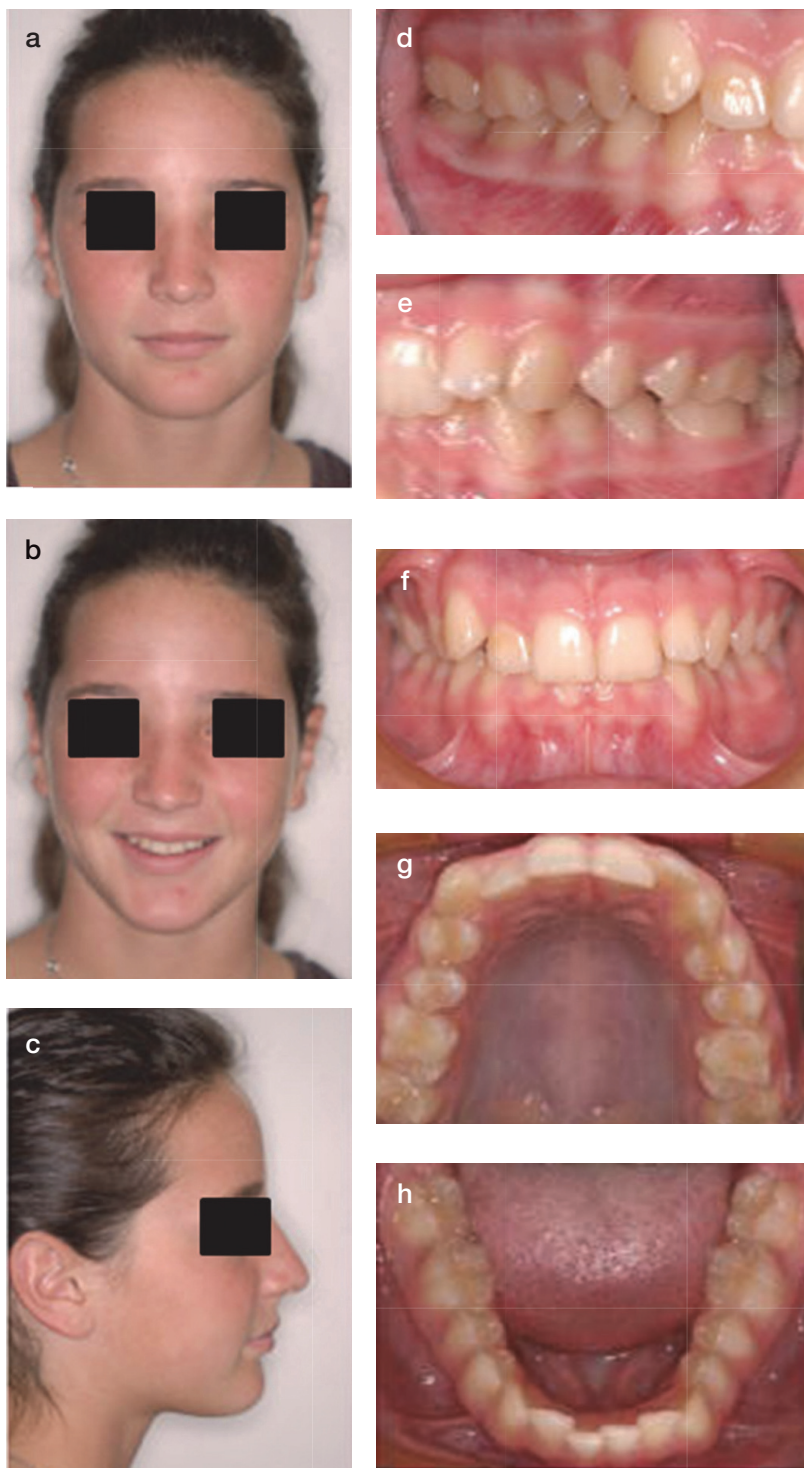

Figure 9 (a-h): Case Report 4, pre-treatment photographs.

lower jaw was further advanced in increments of $1-2 \mathrm{~mm}$. to achieve an edge-to-edge bite. After 13 months, TMJ radiographs were taken to confirm that the condyles were centred in the fossae and subsequently MARA elbows were removed from the left and right sides. The remaining components of the MARA were removed after a fortnight

\section{DIGITIZED LATERAL CEPH, PRE-TREATMENT}

DIGITIZED LATERAL CEPH, POST-TREATMENT

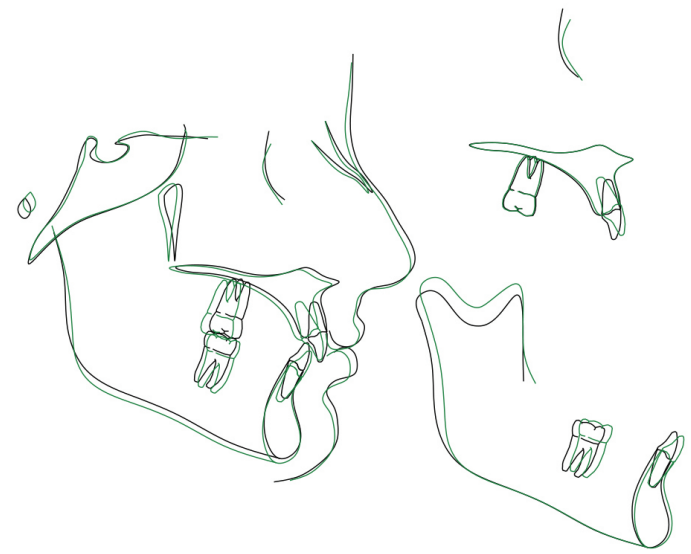

Figure 11: Case Report 4, Ricketts Superimposition - Post-treatment.
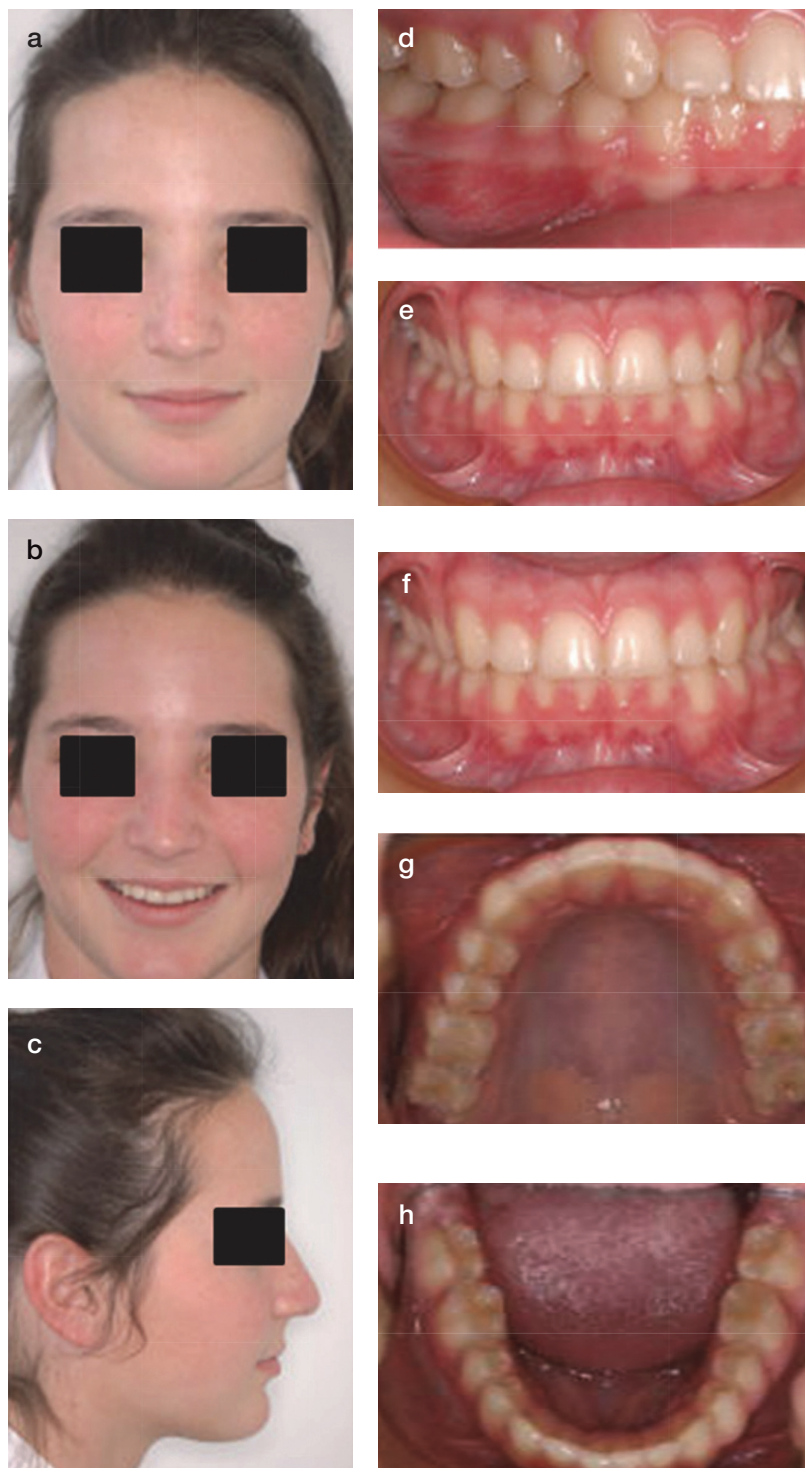

Figure 10 (a-h): Case Report 4, post-treatment photographs.

and final detailing was done to produce a mutually protected functional occlusion. Total treatment time was 22,5 months (Figures 10a-h).

Cephalometric analysis revealed correction of the upper incisor inclination to $24^{\circ}$, the lowers to $26^{\circ}$ and the interincisal angle was reduced to $127^{\circ}$. The ANB angle had improved by a reduction of $3^{\circ}$ with evidence of an increase in mandibular length by 2,5 $\mathrm{mm}$ (Figure 11).

\section{DISCUSSION}

The Clark Twin Block appliance $^{6} \quad$ (Figure 12) that has been used in two of the cases presented

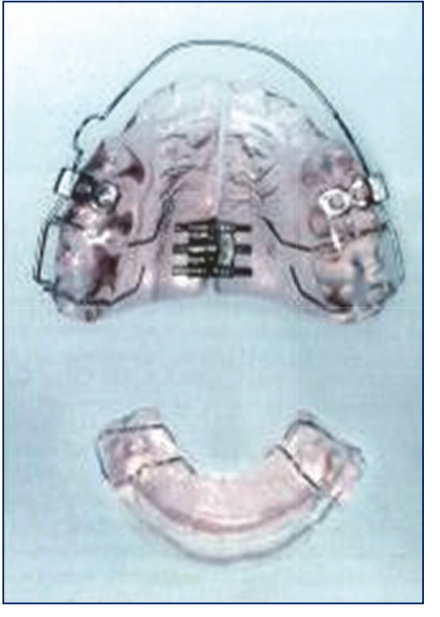

Figure 12: Clark's twin block appliance ${ }^{3}$ here is a modern, highly successful functional appliance modelled after the Schwarz double plate. ${ }^{7}$ This appliance was developed more than 30 years ago by William Clark, and is composed of maxillary and mandibular removable 
acrylic components that fit tightly against the teeth, alveolus and adjacent supporting structures. Treatment with the appliance includes occlusal guide planes, selective grinding of the acrylic, guided eruption and a midpalatal jackscrew to allow for three-dimensional control.

An impeding factor to the success of this treatment modality is patient cooperation and compliance. It is essential that the appliances are worn all night and for most of the day. The latter may be onerous with regard to speech and communication at school. Fortunately the two cases presented were successfully treated without any incident.

Those advocating early treatment believe that the tissues of the craniofacial complex may be more adaptive at a younger age, and pre-adolescent patients may be more compliant than teenagers. ${ }^{10}$

In their investigation of the influences of a fixed functional appliance, Ghislanzoni et al. evaluated the treatment and post-treatment dento-skeletal effects induced by the MARA in the treatment of Class II malocclusion. From this controlled clinical study it was concluded that when compared with matched untreated Class II controls, the cases treated by the MARA showed favourable skeletal changes (mandibular elongation, maxillary growth restriction, ANB decrease, dentoalveolar changes, overjet

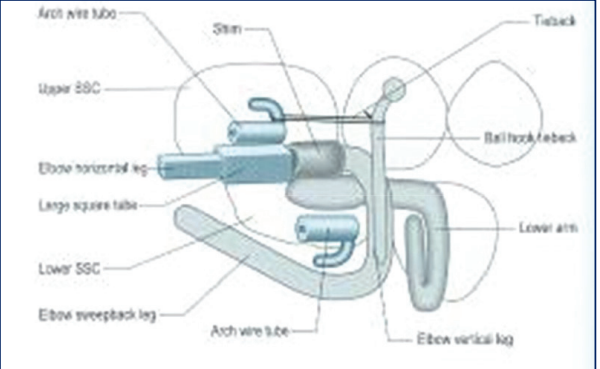

Figure 13a: Components of the MARA ${ }^{8}$

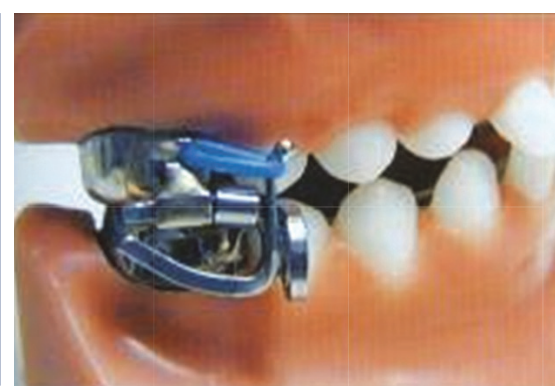

Figure 13b: Intra-oral view of the MARA8 and overbite decrease and correction of molar relationship) that were maintained at an average 1-year post-treatment observation. Furthermore lower incisor proclination is limited, probably as a result of the concurrent use of fixed appliance treatment with MARA.

O'Brian et al. conducted a study to evaluate the effectiveness of early orthodontic treatment

To alleviate the abovementioned restrictions to Class II corrective therapy, the Mandibular Anterior Repositioning Appliance (MARA) (Figures 13a \& 13b) can be employed. Although not as bulky, it can be considered to be a fixed Twin Block, in that it is cemented to the molar teeth and also repositions the lower jaw forward upon closing. The appliance fits around the upper and lower first molars and has two buccally positioned vertical surfaces which contact to keep the lower jaw forward. By holding the lower jaw forward over a sufficiently long period, growth and/or remodelling of the jaws and migration of the teeth can result in a permanent change in the bite from Class II to Class $1 .{ }^{8}$

Functional jaw orthopaedic appliances have a common denominator. All induce a forward mandibular posturing as part of the overall treatment effect, that is, working towards achieving a Class I occlusion during the pivotal growth period.

Controversy exists around the optimal timing for treatment of children with Class II malocclusions. Some believe that treatment should start in the mixed dentition, and others are convinced that early treatment is a waste of time and resources.

There are two general strategies prevailing today regarding the timing of treatment for Class II malocclusion. The first calls for intervention during the pre-adolescent years (ages 8-11) with limited goals that include correction of the molar distocclusion, improvement of the overjet/ overbite relationship and incisor alignment. This so-called "early treatment" is usually followed by a more definitive intervention during adolescence (ages 12-15), which is designed to finish and detail the occlusion. The second major approach to the timing of Class II treatment is not to intervene early and to accomplish the entire correction during the adolescent years. ${ }^{9}$ with the twin-block appliance for the treatment of Class II Division 1 malocclusion. They found that twinblock treatment when a child is 8 to 9 years old has no advantages over treatment started at an average age of 12.4 years. $^{7}$

In another study, Tulloch et al. found that two-phase treatment, started before adolescence in the mixed dentition, might be no more clinically effective than singlephase treatment started during adolescence in the early permanent dentition. ${ }^{9}$

It is evident from this that the optimal timing for treatment of children with Class II malocclusions still remains controversial. Four cases have been presented in the endeavour to demonstrate and support the effectiveness of functional jaw orthopaedics with both the removable (Twin block) and fixed (MARA), functional appliances, as applied in the Department of Orthodontics at the University of Pretoria.

\section{CONCLUSION}

Several appliances can be used in the functional treatment of Class II malocclusions. ${ }^{11,12,13}$ After a comprehensive clinical examination aided by the necessary diagnostic records, the clinician should identify the components that make unique an individual patient's malocclusion. Once this has been established, the appropriate appliance can be selected that would best manage the problem.

Successful orthodontic treatment is evaluated in terms of facial balance, aesthetic harmony and functional stability. The four cases presented offer confirmation that functional jaw orthopaedics can be beneficial. Patients in their pubertal growth should be given an opportunity for early aesthetic improvement with orthopaedic correction. 
The authors wish to thank Prof. P. Botha and Drs. P. Ferreira and B. van Niekerk for supervising the treatment of these patients.

\section{References}

1. King GJ, Keeling SD, Hocevar RA, Wheeler TT. The timing of treatment for Class II malocclusions in children: A literature review. Angle Orthodontics 1990; 2:87-97.

2. Clark WJ, Twin Block Functional Therapy: Appliances in Dentofacial Orthopaedics, 2nd Edition, Mosby Inc. 2002.

3. Ghislanzoni L, Toll DE, Defraia E, Baccetti T, Franchi L. Treatment, post-treatment outcomes induced by the mandibular advancement repositioning appliance: a controlled clinical study. Angle Orthodontist 204; 81:684-91.

4. Graber T, Vandarsdall RL, Vig KWL. Orthodontics: Current Principles and Techniques, 5th Edition, Mosby Inc. 2012.

5. Graber TM, Rakos T, Petrovic A. Dentofacial Orthopedics and Functional Appliances, 2nd Edition, Mosby, 1997.

6. Clark W. Design and management of Twin Blocks: reflections after 30 years of clinical use. J Orthod. 2010; 37(3):209-16.

7. O'Brian K, Wright J, Conboy F, Appelbe P, Davies L, et al. Early treatment for Class II Division 1 malocclusion with the TwinBlock appliance: A multicenter, randomized, controlled trial. Am J Orthod Dentofacial Orthop 2009, 135:573-9.

8. Papadopoulos MA. Orthodontic Treatment of the Class II Non-compliant patient. In: Graber T, Vandarsdall RL, Vig KWL. Orthodontics: Current Principles and Techniques, Mosby Inc. 2006.

9. Tulloch JFC, Proffit WR, Phillips C. Outcomes in a two-phase randomised clinical trial of early Class II treatment. Am J Orthod Dentofacial Orthop 2004; 125:657-67.
10. Ball G, Woodside D, Thompson B, Stuart Hunter W, Posluns J. Relationship between cervical vertebral maturation and mandibular growth. Am J Orthod Dentofacial Orthod 2011; 139:455-61.

11. Proffit WR, Fields HW Jr., Sarver DM. Contemporary Orthodontics. 4th Edition. Mosby Year Book, 2007.

12. Van Beek H, Overjet correlation by a combined headgear and activator. Eur J Orthod 1982; 4:279-90.

13. Clark WJ Twin Block Functional Therapy: Applications in Dentofacial Orthopaedics, 2nd Edition, Mosby Inc, 2002.

\section{6) Gy Gy JONUSINNEWZEALANDANDHAVEA LEESTMETOENW The Dentists love your smile}

\section{NEW ZEALAND PASSENGER ARRIVAL CARD}

Rated in the top ten safest countries in the world - New Zealand is the place where you can enjoy beaches, parks, award-winning wine, world-class restaurants and a range of cultures right on your doorstep - literally.

New Zealand has that perfect combination of big city and country town all rolled into one. With a great community feel, excellent schooling and a thriving economy - New Zealand really is a place that you can call home.

With 91 practices nationwide there are opportunities everywhere to enjoy the lifestyle you want, plus enjoy:

(D) Excellent salary, standard of living and work/life balance

(1) Lucrative earning potential in smaller towns

() Career advancement and development within the Lumino Group

1 We need talented

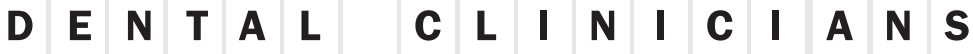

to start

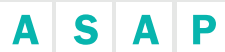

2 For further information:

\begin{tabular}{llllll|l|l|l|l|l|l|l|l|l|l|l|l|l|l|l} 
C & A & $\mathbf{R}$ & $\mathbf{E}$ & $\mathbf{E}$ & $\mathbf{R}$ & $\mathbf{S}$ &. & $\mathbf{L}$ & $\mathbf{U}$ & $\mathbf{M}$ & $\mathbf{I}$ & $\mathbf{N}$ & $\mathbf{0}$ &. & $\mathbf{C}$ & $\mathbf{0}$ &. & $\mathbf{N}$ & $\mathbf{Z}$
\end{tabular} 